\title{
Deciding what information is necessary: do patients with advanced cancer want to know all the details?
}

This article was published in the following Dove Press journal:

Cancer Management and Research

23 May 20II

Number of times this article has been viewed

\section{Bethany J Russell \\ Alicia MWard}

Southern Adelaide Palliative Services, Repatriation General Hospital, Daw Park, SA, Australia
Correspondence: Bethany Russell Southern Adelaide Palliative Services, Repatriation General Hospital, 700 Goodwood Road, Daw Park, SA 504I, Australia

Tel +6I 882751732

Fax +6I 882774957

Email bethany.russell@health.sa.gov.au

\begin{abstract}
Communicating effectively with patients who have advanced cancer is one of the greatest challenges facing physicians today. Whilst guiding the patient through complex diagnostic and staging techniques, treatment regimens and trials, the physician must translate often imprecise or conflicting data into meaningful personalized information that empowers the patient to make decisions about their life and body. This requires understanding, compassion, patience, and skill. This narrative literature review explores current communication practices, information preferences of oncology patients and their families, and communication strategies that may assist in these delicate interactions. Overwhelmingly, the literature suggests that whilst the majority of patients with advanced cancer do want to know their diagnosis and receive detailed prognostic information, this varies not only between individuals but also for a given individual over time. Barriers to the delivery and understanding of information exist on both sides of the physician-patient relationship, and family dynamics are also influential. Despite identifiable trends, the information preferences of a particular patient cannot be reliably predicted by demographic, cultural, or cancer-specific factors. Therefore, our primary recommendation is that the physician regularly asks the patient what information they would like to know, who else should be given the information and be involved in decision making, and how that information should be presented.
\end{abstract}

Keywords: metastatic, communication, prognosis, physician-patient relations

\section{Introduction}

Physicians caring for patients with advanced cancer are increasingly expected to not only remain up to date with the latest diagnostic and staging modalities, treatment regimens and trials, but to also communicate effectively with patients and families regarding these matters. The conversations that punctuate each patient's cancer journey are technically complex and have intense social, psychological, and spiritual significance, ${ }^{1}$ requiring understanding, compassion, patience, and skill. The aim of this narrative literature review is to explore current communication practices and how both physician and patient factors influence these, how patients interpret and use the information communicated to them, and patient preferences for the delivery of information. Strategies and guidelines for conducting such delicate conversations and the role of training are also discussed.

A literature search using PubMed and the terms 'advanced cancer/malignancy', 'metastatic cancer/malignancy' or 'terminal cancer/malignancy' and 'communication' was conducted. Hand-searching of reference lists of relevant articles was also performed. English-language qualitative and quantitative studies have been included. 
As doctor-patient communication is the focus of this review, the nursing and allied health literature was not specifically searched. This paper is written from a Western perspective, and as most of the literature retrieved pertains to disclosing diagnosis and discussing prognosis, this is the main focus of the review.

\section{Current communication practices}

Communication with oncology patients has changed dramatically over the last 50 years. While previously the very diagnosis of cancer was withheld from most patients, it is now almost always disclosed, ${ }^{2,3}$ although in non-Western countries this may not be common practice. ${ }^{4-7}$ The survival implication of that diagnosis however - the prognosis - remains poorly communicated. A study of 140 patients with metastatic cancer in the Netherlands found only 39\% were told their prognosis by their oncologist. ${ }^{8}$ Similarly, an Australian study of patients with breast cancer or melanoma found only $27 \%$ were given a prognosis during their initial oncology consultation, while $57 \%$ desired this information. ${ }^{9}$

Oncology patients generally tend to overestimate their prognosis. In the Study to Understand Prognoses and Preferences for Outcomes and Risks of Treatments (SUPPORT), 82\% of 917 patients hospitalized with metastatic cancer in the United States estimated their 6-month survival more optimistically than their physician, and in over half of this was by a factor of $40 \%$ or more. ${ }^{10}$ Other studies have demonstrated high rates of confusion in advanced cancer patients as to whether their cancer is curable or incurable. ${ }^{11,12}$ There are also high rates of discordance between patients and their families, with one Australian survey finding in over a third of those with incurable cancer only one of the patient-carer pair understood that their treatment was not intended to cure. ${ }^{13}$

Patient-physician disparity regarding understanding of prognosis may be due to a range of factors (Table 1).

\section{Physician factors}

Firstly, the physician may choose not to disclose prognostic information. In many cases, life expectancy is only discussed if the patient asks, and in some cases not even if requested. ${ }^{14,15}$ In a large American survey, while physicians felt able to formulate a prognosis for $96 \%$ of their advanced cancer patients, they stated they would only communicate this prognosis to $37 \%$, even if a survival estimate were requested. They would not communicate any survival estimate to $22 \%$ of patients and would communicate a different estimate to the one they had formulated to $40 \%$, of
Table I Factors that may contribute to doctor-patient disparity in understanding of prognosis

\begin{tabular}{ll}
\hline Physician factors & Patient factors \\
\hline Reluctance to disclose prognosis & Ambiguous attitude to \\
Low confidence in ability & knowing prognosis \\
to prognosticate accurately & Denial \\
Low confidence in ability to & Distress \\
discuss prognosis & Preferring to entrust details \\
Insufficient time & to experts \\
Fear of destroying hope & Fear of causing offense by \\
Fear of provoking emotional & questioning \\
distress & Fear of wasting the physician's \\
Fear of being blamed & time \\
Fear of confronting own emotions & Fear of losing hope \\
Fear of confronting death & Cultural expectations \\
Feelings of inadequacy & Difficulty understanding \\
Burnout and compassion fatigue & terminology or certain \\
Cultural expectations & information formats \\
Overestimation of patient & \\
understanding & \\
\hline
\end{tabular}

which $70 \%$ would be optimistically disparate. ${ }^{16}$ If patients are optimistic then many physicians reinforce optimistic views. Most avoid trying to be specific when asked for prognostic information, particularly if generalists compared with specialists. ${ }^{14}$

The reluctance of physicians to disclose prognostic information may be due to low confidence in their ability to prognosticate accurately. As effective therapies for previously fatal diseases were discovered, medical education and literature have increasingly focused on diagnosis and treatment, with a resultant loss of the skill of prognostication. ${ }^{17}$ Many physicians therefore find prognostication difficult and stressful, and feel inadequately trained to prognosticate. ${ }^{14}$

It is true that prognostic estimates in terminally ill patients are often incorrect and usually optimistic, ${ }^{18-22}$ with one report of estimates being over three times longer than actual survival. ${ }^{16}$ The experience and specialty of physician as well as the nature of the physician-patient relationship can influence the accuracy of a clinical prediction of survival. ${ }^{19,23}$ Accuracy may improve closer to death ${ }^{20,21}$ and with the addition of calculated prognostic indices such as the palliative prognostic index. ${ }^{24}$

Physicians may also feel they have inadequate skills or training to discuss prognosis. They may fear destroying hope or provoking emotional distress. They may feel they have inadequate training or time to attend to a patient's emotional needs, and may fear being blamed by patients and relatives. They may have feelings of inadequacy or hopelessness regarding the unavailability of further curative treatment, 
may worry about containing their own emotions, and may have to confront their own fears about death. ${ }^{14,25-29}$ Clinicians who care for dying patients are also at risk of burnout and compassion fatigue, which may lead to emotional exhaustion, diminished empathy, and avoidance of emotionally difficult clinical situations. ${ }^{30}$

Physicians' concerns about discussing prognosis may be well founded. Patients can be traumatized when bad news is delivered bluntly, ${ }^{31}$ and major changes in prognosis may be destabilizing. ${ }^{32}$ However, if potentially distressing information is withheld from patients then they are given no opportunities to reveal their own fears and worries. They may feel isolated and worried that nothing will be done to help, and may imagine a more horrible outcome than awaits them. As Fallowfield asserts, "precisely when the majority of people are most in need of truthful communication and support, when they have changing thoughts and feelings and need to make important decisions, a conspiracy of silence may envelop them and the resulting anxiety and tension may hinder adjustment". ${ }^{29}$

Conversely, prognostic information that is tailored to a patient's preferences has potential to improve their psychological wellbeing. ${ }^{1,33}$ Tailored prognostic information has been found to result in higher satisfaction with care, lower anxiety and depression scores, and even improved cognitive function. ${ }^{34-36}$ Potentially distressing information delivered sensitively can be used to reassure by allowing planning around symptom progression and actual mode of death, such as preparing specific crisis orders for catastrophic hemorrhage. ${ }^{37,38}$

Cultural elements also influence physician communication. A study of palliative medicine physicians showed those from South America and Europe were far less likely than their Canadian counterparts to believe that the majority of their patients wanted to know about the terminal stage of their illness; $18 \%$ and $26 \%$ versus $93 \%$, respectively. ${ }^{3}$ Oncologists practicing in non-Western countries may be more likely to wait for the patient to ask before disclosing a poor prognosis. ${ }^{27}$

\section{Patient factors}

Another reason there may be disparity in patient-physician understanding of prognosis is that the physician may be discouraged from disclosing information either by the patient or by their family. The phenomenon of collusion has been described by oncologist and ethicist Paul Helft as a "spoken or unspoken agreement" between the physician and patient, entered into "to avoid or delay discussing a definitive, numerical prognostic estimate of life expectancy". ${ }^{39}$ Patients with terminal cancer have identified a sense of ambiguity regarding prognostic information, wherein they want to be told but simultaneously do not want to know. ${ }^{40}$ Discussion may tend to focus around the treatment calendar and ignore the long term. This approach can provide optimism, which helps the patient to endure the treatment phase but may lead to regret later. ${ }^{41}$

Reasons why patients may choose not to ask their physician questions about their malignancy and treatment may include faith in the physician as an expert and hence relief in not being required to understand the details personally, fear of causing offence by questioning, and not wanting to waste the physician's time with questions when other patients are waiting. Additionally, they may not ask questions in an attempt to preserve hope and present a 'brave face', although in some patients, preserving hope conversely translates to more active information seeking, particularly regarding alternative therapies. ${ }^{32}$

In cultures where knowledge of diagnosis and prognosis is thought to be detrimental to the patient's wellbeing, family members may prefer the physician not to speak frankly with the patient. ${ }^{42-44}$ Oncologists practicing in non-Western countries may be more likely to comply with such family requests. ${ }^{27}$

\section{How patients interpret what is said}

Lastly, there may be patient-physician disparity in the understanding of prognosis if patients have misunderstood the information that has been provided. Medical terminology and various formats of presenting prognosis and probabilities can be difficult for patients to comprehend. ${ }^{45,46}$ Words that carry different meanings in nonmedical conversation, such as "progressing" and "positive/negative" tests, may cause confusion. ${ }^{47-49}$ Distress and denial are known to reduce the amount of information patients retain after conversations with physicians. ${ }^{50,51}$ Data from the Australian and American contexts have shown that age and gender do not appear to be significant factors in comprehension, but patients who have not completed secondary school and for whom English is a second language are less likely to have concordance with their physician. ${ }^{51,52}$

Physicians may overestimate patient understanding, ${ }^{53}$ and Australian oncologists have been reported to seek confirmation of understanding in only $10 \%$ of consultations. ${ }^{35}$ Patients may be reluctant to ask for clarification, particularly when there is a language barrier. ${ }^{54}$

\section{How information influences decision-making}

Regardless of the reliability of the information gleaned, patients and their families do use it to make important decisions regarding the nature of their treatment, estate management, 
and funeral planning, in addition to social planning including summoning relatives to visit from out of town. ${ }^{45,55}$ When the information is inaccurate or based on hopes rather than facts, their decisions may not reflect their true values. Similarly, overly optimistic prognostic estimates may influence treatment decisions made by physicians, with resultant futile interventions such as chemotherapy in the last few weeks of life, no discussion of palliative care, and late referrals to hospice. ${ }^{56}$

In the SUPPORT study, cancer patients who were over $90 \%$ sure they would live for at least 6 months were over two and a half times more likely to favor life-extending therapy over symptom-focused care compared with those who thought there was even a $10 \%$ chance they would not live 6 months. Those preferring life-extending therapy were more likely to undergo aggressive therapy, but their 6-month survival was no better than the more pessimistic group. Instead they were more likely to have a readmission to hospital, an attempted resuscitation, or a death whilst receiving ventilatory assistance. ${ }^{10}$ Another American study found patients who were aware they are terminally ill were more likely to discuss endof-life care with their physician and more likely to receive care that was consistent with their wishes. Importantly, patients with advanced cancer who received life-extending care in the last week of life were rated by caregivers as having higher physical and psychological distress and poorer quality of life than those who received symptom-directed care, regardless of the patient's treatment preferences. ${ }^{57}$ End-of-life discussions have also been associated with lower medical costs in the final week of life. ${ }^{58}$ Discussions about no further curative treatment and transition to hospice care, however, have been reported by American oncologists to be those they find most difficult. $^{27}$

Both patients and carers identify psychosocial preparation for death as an important part of a 'good death'. This includes saying goodbye to important people, resolving unfinished business matters and having a general sense of completion. Undergoing aggressive therapies in the last days and weeks of life may reduce the patient's ability and opportunities to engage in these activities, which take time and may be both physically and psychologically taxing. ${ }^{59}$ Understanding prognosis has implications for carers also, with evidence of preparedness for the death of a loved one decreasing long-term anxiety rates and facilitating grief resolution. ${ }^{60,61}$

The literature suggests, therefore, that due to a lack of disclosure or subsequent misunderstanding of information disclosed, physicians and patients often do not share the same understanding of the patient's prognosis. Given the potentially profound influence of this information on patient decision making around not only treatment but social and financial matters, it is crucial for physicians to ensure that the patient has opportunity to receive and comprehend accurate, clear, and personalized information. To achieve this goal, physicians need to know which pieces of information to convey, how to convey them, and when.

\section{Patient preferences for information delivery Content and timing: what patients want to hear and when}

Over one-third of patients with advanced cancer feel that they have high unmet needs with respect to medical information and communication. ${ }^{62}$ The majority of oncology patients indicate a desire to know their diagnosis and the extent of disease, their treatment options, and even relatively rare side effects of these treatments. ${ }^{63,64}$

Most oncology patients also want to know their prognosis $^{47,64}$ and have rated prognostic information as the most important element of communication-more important than diagnostic disclosure or treatment information. ${ }^{65} \mathrm{~A}$ large UK study of 2850 patients with cancer of various clinical stages showed $85 \%$ wanted the doctor to provide as much information as possible. ${ }^{47} \mathrm{~A}$ desire for information may not be true for all patients or all cultural groups however.,47 Equally, a patient's desire for information is not always accompanied by a desire to be involved in clinical decision making, as some find this burdensome. ${ }^{32,66}$

Oncology patients want physicians to communicate honestly with them about the severity of their disease ${ }^{40,46,67}$ but to also be optimistic. ${ }^{38,68} \mathrm{In}$ a study of Australian women with metastatic breast cancer, Butow and colleagues summarized that when these women do request prognostic information "they are rarely seeking statistics on life expectancy, but rather reassurance and hope, forewarnings concerning the impact of side effects and symptoms on their lives, and/ or guidance regarding practical decisions they may need to make". ${ }^{69}$

Evidence suggests that female patients, ${ }^{47,66}$ those with better education, ${ }^{70}$ and those in the first six decades of life may desire more information than their counterparts..$^{36,47}$ Certain types of cancer may also correlate with higher informational needs; for example, ovarian as compared to breast. ${ }^{70}$ Higher depression scores are associated with wanting to know the shortest time to live without treatment. ${ }^{64}$

Despite these trends, it is important to note that demographics do not reliably predict individual informational preferences, and studies have found contradicting results. ${ }^{9,71}$ Physicians 
must attempt to engage with their patients on a personal level, taking into account their unique character, experiences, and social setting, as well as their fears and hopes.

Many patients want to negotiate if and when expected survival is discussed, ${ }^{64,69,72}$ and there is variation in patients' beliefs as to who should initiate these conversations. ${ }^{50}$ Patients have indicated that physicians should not assume they wish to be told prognosis but permission to discuss should be sought periodically, ${ }^{64}$ although even this is not a simple matter. When American patients with either advanced cancer or severe chronic obstructive pulmonary disease and their families were asked "how much information do you want?", all initially replied that they wanted "all the information" but detailed interviews subsequently revealed variation in desire and readiness for explicit prognostic information. ${ }^{73}$ Information, decision making, and indeed care preferences may fluctuate over the course of an illness and may also differ between patients and their carers. ${ }^{32,66,74}$

Australian research suggests that caregivers more than patients themselves want details regarding the terminal phase. Patients are keen to know that pain will be controlled as their disease progresses, but for other symptoms, they only want reassurance of support if and when they arise. In contrast, caregivers request detailed information about future symptoms, mode of death, and whether caring for their loved one at home would be a realistic option. ${ }^{75}$

Some evidence suggests a general trend of patients desiring less detail as time goes on. ${ }^{66}$ Other studies show informational needs of patients and their families diverging as cancer progresses, with patients becoming more focused on symptom management and family remaining interested in prognosis. Patients and family members may shelter each other from knowledge as the illness progresses, with communication between them becoming less verbally explicit. ${ }^{40}$ Conversely, the communication style of relatives towards people other than the patient becomes more open and explicit, particularly about dying itself, as the death of the patient draws near. ${ }^{76}$

\section{Key players: who patients want to be involved}

Patients want their prognostic information to be delivered by an expert physician who is directly in charge of their care ${ }^{40}$ and preferably a physician who they trust, who sees them as a whole person, and who is comfortable talking about death and the patient's fears. ${ }^{59,46}$ Patients are more likely to perceive information given as unclear when delivered by a house medical officer compared with a specialist or general practitioner, reflecting the importance of experience and training. ${ }^{43}$ There is conflicting data as to patient preferences for having a nurse or other health professional accompany them during consultations, ${ }^{9}$ although ensuring a team approach and minimizing the number of people presenting information may help to avoid exposure to contradictory information, which provokes anxiety. ${ }^{32,71}$

Family members frequently provide significant emotional support along the patient's cancer journey and help them to remain hopeful, ${ }^{77}$ but not all patients desire a family member to be present when receiving new medical information. ${ }^{9}$ While most patients find this a source of support, others want to be at liberty to discuss sensitive issues with their physician without burdening family and to filter information for their family members. ${ }^{50}$ The majority of oncology patients want family members to be involved in decision making but not necessarily at all points of their illness. ${ }^{78}$

Cultural issues may influence the degree to which oncology patients want their families to be involved. ${ }^{6,79}$ Many non-Western and indigenous cultures have a family-centered model of medical decision making, rather than a patient autonomy model. ${ }^{80,81}$ They may believe family members should have access to accurate and honest information regarding the patient's diagnosis and prognosis, but this should be withheld from the patient. They may also believe that family rather than the patient should make decisions about treatment; including the use of life support. Thus, well meaning attempts to protect the autonomy of the individual, with advanced care directives for example, may actually oppose their deepest values. ${ }^{81}$

\section{Style of delivery: verbal and nonverbal aspects}

The communication style of the physician influences the content and nature of patients' responses and their willingness to raise issues which are important to them. Acknowledging emotion is a central component of showing empathy and facilitating discussion about patients' concerns, which may ameliorate distress even if the problems themselves cannot be solved. ${ }^{82}$ Oncology patients do not often express negative emotions to their oncologists ${ }^{83}$ but report an increased need to talk about their worries and fears. ${ }^{66}$ Audio-recordings of consultations in America have found empathic opportunities are present in 37\% of conversations, but oncologists give empathic responses less than one-third of the time; a low rate which likely discourages any further expression of emotion by patients. ${ }^{84}$ Similar rates have been found among nonphysician clinicians such as nurse practitioners and physician assistants. ${ }^{85}$

Many patients with advanced cancer want their physician to shift from service provider to ally. They fear that they 
will be medically uninteresting and less rewarding for their physicians but want them to continue their commitment and provide options for care, albeit palliative. ${ }^{72}$ Patients want to be acknowledged as individuals, given an opportunity to ask questions and be asked at regular intervals what their information preferences are. ${ }^{32,77}$

Many patients do not want to be told a timeframe, but if prognosis is discussed directly, most desire this to be in the format of how long the average person with their condition would live. ${ }^{86}$ Patients generally prefer positively presented information such as "chance of living $\times$ years" rather than "chance of dying $\times$ time" ${ }^{64}$ Making just one pessimistic statement during the consultation has been shown to ensure effective communication of a poor prognosis, but physicians can simultaneously emphasize optimistic aspects of the patient's case to foster hope. ${ }^{87}$ Other strategies to maintain hope include setting achievable goals, emphasizing symptomatic therapies that can be offered, and reassuring that there will be continuity of care and support throughout the course of the patient's disease. ${ }^{40,75,77,88}$

Patients also report that words and numbers are perceived to be more optimistic and less harsh than pie charts and graphs. ${ }^{64}$ Up to $80 \%$ appreciate the occasional use of humor but feel the use of euphemisms (eg, 'pass away' rather than 'die') do not facilitate hope. ${ }^{77}$ The use of metaphors and analogies however (eg, likening a chemotherapy regimen to a marathon) can improve patient understanding and satisfaction with communication, due to the incorporation of everyday concepts that are more familiar to patients than medical terminology ${ }^{89}$

Where language barriers exist, some patients prefer translation to be performed by family members who are trusted and accessible, while others prefer professionals who aren't emotionally invested in the conversation. ${ }^{54,71}$ 'Cultural interpreters', such as Aboriginal health workers, may also be helpful in providing guidance in cultural norms and facilitating understanding of illness concepts that may be underpinned by completely different worldviews. ${ }^{90,91}$

Nonverbal communication is also important. The vast majority of oncology patients prefer face-to-face communication, ${ }^{9}$ and randomized controlled trials of physicians' posture whilst breaking bad news have shown patients prefer physicians to sit rather than stand, and perceive those that do so as more compassionate. ${ }^{92,93}$ Patients with advanced cancer perceive physician behaviors such as making eye contact (if culturally appropriate) and not taking phone calls to convey a sense of being present and not hurried. This is felt to be more important than the actual amount of time spent. Some patients appreciate physical touch and sitting on the patient's bed rather than on a chair, but hugging is generally considered to be outside professional boundaries. ${ }^{72,94}$

The concept of using a 'treatment broker' has been suggested, where a third party who is trusted by both the doctor and patient assists both to clarify their understanding, assumptions, and expectations. ${ }^{41}$ Family members may often informally fulfill this role, though specialized nursing or support staff who can remain objective may best perform it. Addition of written communications and audiocassette recording of the consultation improves recall of the information given and overall patient satisfaction. ${ }^{95}$ Provision of a question prompt list before a palliative medicine consultation results in patients asking twice as many questions and more discussion of prognostic and end-of-life issues, although may not lower patient anxiety levels or improve their satisfaction scores. ${ }^{96}$

Alternative sources of information, such as books, pamphlets, broadcast media, telephone services, and the Internet, have been found to decrease uncertainty and give some sense of control, particularly for younger patients or when communication with the health professional was felt to be unsatisfactory. ${ }^{40,70}$ Immigrants appreciate these resources when available in their native language.${ }^{54}$ Physician recommendation of particular reliable websites is appreciated. ${ }^{72}$ Patients have reported frustration and anxiety with nonindividualized materials, however, in which they struggle to discern which information is relevant to their own case. ${ }^{32}$ It is also important to note that patients, particularly women, seek information from one another with regards to personal experiences of cancer and cancer treatment. ${ }^{32}$

\section{Improving communication skills}

Good communication is essential for health professionals as it improves health care outcomes and has been shown to prevent burnout and increase job satisfaction. ${ }^{97,98}$ Increased skills and confidence in discussing poor prognostic information with patients has been reported by physicians who have undertaken training in the 'SPIKES' protocol' ${ }^{99}$ and workshops such as 'Oncotalk' ${ }^{100}$ A Cochrane systematic review concluded that communication skills do not reliably improve with experience alone, but that there is some evidence from three studies to suggest intensive multiday or modular communications skills training can have beneficial effects on communication practices in professionals working with cancer patients. It is not clear if this is reproducible for other training facilitators or participants. ${ }^{101}$ Subsequently published results of a 12-month follow-up of 160 oncologists showed enduring effects over time. ${ }^{98}$ Such courses, however, are expensive and require a significant time commitment. 
Promising results have also been seen with a novel training approach using computer simulated standardized patients, although these lack nonverbal communication and emotional cues, which are so essential in the real world. ${ }^{102}$

While available training courses are often directed at oncologists and oncology fellows, many patients have their diagnosis of cancer communicated by general practitioners or surgeons. ${ }^{96,103}$ General practitioners are often also used as a sounding board for decisions and to verify information given by other physicians. Hence there is a need for medical schools and specialty colleges to include communication skills training in their core curricula. Clinical practice guidelines ${ }^{1,71}$ provide all clinicians caring for patients with cancer and their families with evidence-based, practical approaches for conducting difficult conversations.

Further research is required into communication with oncology patients and their families, as well as the most effective method of teaching clinicians how to communicate effectively in keeping with patient preferences. Training courses must result in skill development that is transferrable to the everyday clinical setting and that endures over time.

\section{Summary}

Overwhelmingly, the literature suggests that whilst the majority of oncology patients do want detailed information, this varies not only between individuals, but also for a given individual over time. This narrative review highlights trends emerging from existing evidence, but even so, the information preferences of an individual cannot be reliably predicted by either demographic or cancer-specific factors.

Communication between the physician and oncology patient is highly contextualized and complex. Therefore, our primary recommendation is that the physician regularly asks the patient what information they would like to know, who else should be given the information and be involved in decision making, and also how that information should be presented.

\section{Acknowledgments}

The authors wish to thank Professor David Currow, Dr Timothy To, and Dr Sally Williams for their advice and assistance in preparing this manuscript.

\section{Disclosure}

The authors declare no competing interests.

\section{References}

1. National Breast Cancer Centre and National Cancer Control Initiative Clinical practice guidelines for the psychosocial care of adults with cancer. Camperdown, NSW: National Breast Cancer Centre; 2003.
2. Novack DH, Plumer R, Smith RL, Ochitill H, Morrow GR, Bennett JM. Changes in physicians' attitudes toward telling the cancer patient. JAMA. 1979;241(9):897-900.

3. Bruera E, Neumann CM, Mazzocato C, Stiefel F, Sala R. Attitudes and beliefs of palliative care physicians regarding communication with terminally ill cancer patients. Palliat Med. 2000;14(4):287-298.

4. Centeno-Cortes C, Nunez-Olarte JM. Questioning diagnosis disclosure in terminal cancer patients: a prospective study evaluating patients' responses. Palliat Med. 1994;8:39-44.

5. Pronzato P, Bertelli G, Losardo P, Landucci M. What do advanced cancer patients know of their disease? A report from Italy. Support Care Cancer. 1994;2(4):242-244.

6. Gongal R, Vaidya P, Jha R, Rajbhandary O, Watson M. Informing patients about cancer in Nepal: what do people prefer? Palliat Med. 2006;20(4):471-476.

7. Elwyn TS, Fetters MD, Sasaki H, Tsuda T. Responsibility and cancer disclosure in Japan. Soc Sci Med. 2002;54(2):281-293.

8. Koedoot CG, Oort FJ, de Haan RJ, Bakker PJ, de Graeff D, de Haes JC The content and amount of information given by medical oncologists when telling patients with advanced cancer what their treatment options are: palliative chemotherapy and watchful-waiting. Eur J Cancer. 2004;40(2):225-235.

9. Butow PN, Kazemi JN, Beeney LJ, Griffin A, Dunn SM, Tattersall MHN. When the diagnosis is cancer: patient communication experiences and preferences. Cancer. 1996;77(12):2630-2637.

10. Weeks JC, Cook EF, O'Day SJ. Relationship between cancer patients' predictions of prognosis and their treatment preferences. JAMA. 1998;279(21):1709-1714.

11. Mackillop WJ, Stewart WE, Ginsburg AD, Stewart SS. Cancer patients' perceptions of their disease and its treatment. Br J Cancer. 1988;58 (3): 355-358.

12. Beadle GF, Yates PM, Najman JM, et al. Beliefs and practices of patients with advanced cancer: implications for communication. Br J Cancer. 2004;91(2):254-257.

13. Burns CM, Bromm DH, Smith WT, Dear K, Craft PS. Fluctuating awareness of treatment goals among patients and their caregivers: a longitudinal study of a dynamic process. Support Care Cancer. 2007;15(2):187-196.

14. Christakis NA, Iwashyna TJ. Attitude and self-reported practice regarding prognostication in a national sample of internists. Arch Intern Med. 1998;158(21):2389-2395.

15. Michiels E, Deschepper R, Bilsen J, Mortier F, Deliens L. Information disclosure to terminally ill patients and their relatives: self-reported practice of Belgian clinical specialists and general practitioners. Palliat Med. 2009;23(4):345-353.

16. Lamont EB, Christakis NA. Prognostic disclosure to patients with cancer near the end of life. Ann Intern Med. 2001;134(12): 1096-1105.

17. Christakis NA. The ellipsis of prognosis in modern medical thought. Soc Sci Med. 1997;44(3):301-315.

18. Parkes CM. Accuracy of predictions of survival in later stages of cancer. Br Med J. 1972:2(5804):29-31.

19. Christakis NA, Lamont EB. Extent and determinants of error in doctors' prognoses in terminally ill patients: prospective cohort study. $B M J$. 2000;320(7233):469-472.

20. Glare P, Virik K, Jones M, et al. A systematic review of physicians' survival predictions in terminally ill cancer patients. $B M J$. 2003;327(7408):195-198.

21. Fromme EK, Smith MD, Bascom PB, Kenworthy-Heinige T, Lyons $\mathrm{KS}$, Tolle SW. Incorporating routine survival prediction in a US hospital-based palliative care service. J Palliat Med. 2010;13(2): 1439-1444.

22. Kao SC, Butow P, Bray V, Clarke SJ, Vardy J. Patient and oncologist estimates of survival in advanced cancer patients. Psychooncology. 2011;20(2):213-218.

23. Smith JL. Commentary: why do doctors overestimate? BMJ. 2000; 320(7233): 472-473. 
24. Morita T, Tsunoda J, Inoue S, Chihara S. Improved accuracy of physicians' survival prediction for terminally ill cancer patients using the palliative prognostic index. Palliat Med. 2001;15(5):419-424.

25. Buckman R. Breaking bad news: why is it still so difficult? BMJ (Clin Res Ed). 1984;288(6430):1597-1599.

26. Fallowfield LJ. Giving sad and bad news. Lancet. 1993;341:476-478.

27. Baile WF, Lenzi R, Parker PA, Buckman R, Cohen L. Oncologists' attitudes toward and practices in giving bad news: an exploratory study. J Clin Oncol. 2002;20(8):2189-2196.

28. Morita T, Akechi T, Ikenaga M, et al. Communicating about the ending of anticancer treatment and the transition to palliative care. Ann Oncol. 2004;15(10):1551-1557.

29. Fallowfield L. Communication with the patient and family in palliative medicine. In: Doyle D, Hanks G, Cherny N, Calman K, editors. Oxford Textbook of Palliative Medicine. Oxford: Oxford University Press; 2005:101-107.

30. Kearney MK, Weininger RB, Vachon MLS, Harrison RL, Mount BM. Self-care of physicians caring for patients at the end of life. JAMA. 2009;301(11):1155-1164.

31. Bedell SE, Graboys TB, Bedell E, et al. Words that harm, words that heal. Arch Int Med. 2004;164(13):1365-1368.

32. Leydon GM, Boulton M, Moynihan C, et al. Cancer patients; information needs and information seeking behavior: in depth interview study. $B M J$. 2000;320(7239):909-913.

33. Bishara E, Loew F, Forest MI, Fabre J, Rapin CH. Is there a relationship between psychological well-being and patient-carers consensus? A clinical pilot study. J Palliat Care. 1997;13(4):14-22.

34. Schofield PE, Beeney IJ, Thompson JF, Butow PN, Tattersall MH, Dunn SM. Hearing the bad news of a cancer diagnosis: the Australian melanoma patient's perspective. Ann Oncol. 2001;12(3):365-371.

35. Gattellari M, Voight KJ, Butow PN, Tattersall MHN. When the treatment goal is not cure: are cancer patients equipped to make informed decisions? J Clin Oncol. 2002;20(2):503-513.

36. Yun YH, Kwon YC, Lee MK, et al. Experiences and attitudes of patients with terminal cancer and their family caregivers toward the disclosure of terminal illness. J Clin Oncol. 2010;28(11):1950-1957.

37. Barnett MM. Does it hurt to know the worst? - psychological morbidity, information preferences and understanding of prognosis in patients with advanced cancer. Psychooncology. 2006;15(1):44-55.

38. Kutner JS, Steiner JF, Corbett KK, Jahnigen DW, Barton PL. Information needs in terminal illness. Soc Sci Med. 1999;48(10):1341-1352.

39. Helft PR. Necessary collusion: prognostic communication with advanced cancer patients. J Clin Oncol. 2005;23(13):3146-3150.

40. Kirk P, Kirk I, Kristjanson LJ. What do patients receiving palliative care for cancer and their families want to be told? A Canadian and Australian qualitative study. BMJ. 2004;328(7452):1343.

41. The AM, Hak T, Koëter G, ven der Wal G. Collusion in doctor-patient communication about imminent death: an ethnographic study. BMJ. 2000;321(7273):1376-1381.

42. Mitchison D, Butow P, Sze M, et al. Prognostic communication preferences of migrant patients and their relatives. Psychooncology. [Epub ahead of press].

43. Chan A, Woodruff RK. Communicating with patients with advanced cancer. J Palliat Care. 1997;13(3):29-33.

44. Tse CY, Chong A, Fok SY. Breaking bad news: a Chinese perspective. Palliat Med. 2003;17(4):339-343.

45. Lobb EA, Butow PN, Kenny DT, Tattersall MHN. Communicating prognosis in early breast cancer: do women understand the language used? MJA. 1999;171(6):290-294.

46. Wenrich MD, Curtis JR, Shannon SE, et al. Communicating with dying patients within the spectrum of medical care from terminal diagnosis to death. Arch Intern Med. 2001;161(6):868-874.

47. Fallowfield LJ, Jenkins VA, Beveridge HA. Truth may hurt but deceit hurts more: communication in palliative care. Palliat Med. 2002;16(4): 297-303.
48. Chapman K, Abraham C, Jenkins V, Fallowfield L. Lay understanding of terms used in cancer consultations. Psychooncology. 2003;12(6): 557-566.

49. Yardley SJ, Davis CL, Sheldon F. Receiving a diagnosis of lung cancer: patients' interpretations, perceptions and perspectives. Palliat Med. 2001;15(5):379-386.

50. Clayton JM, Butow PN, Psych M, Tattersall MHN. When and how to initiate discussion about prognosis and end-of-life issues with terminally ill patients. J Pain Symptom Manage. 2005;30(2):132-144.

51. Gattellari M, Butow PN, Tattersall MHN, Dunn SM, MacLeod CA. Misunderstanding in cancer patients: Why shoot the messenger? Ann Oncol. 1999;10(1):39-46.

52. DesHarnais S, Carter RE, Hennessy W, Kurent JE, Carter C. Lack of concordance between physician and patient: reports on end-of-life care discussions. J Palliat Med. 2007;10(3):728-740.

53. Quirt CF, Mackillop WJ, Ginsburg AD, et al. Do doctors know when their patients don't? A survey of doctor-patient communication in lung cancer. Lung Cancer. 1997;18(1):1-20.

54. Butow PN, Sze M, Dugal-Beri P, et al. From inside the bubble: migrants' perceptions of communication with the cancer team. Support Care Cancer. 2011;19(2):281-290.

55. Glare PA, Sinclair CT. Palliative medicine review: prognostication. $J$ Palliat Med. 2008;11(1):84-103.

56. Earle CC, Landrum MB, Souza JM, et al. Aggressiveness of cancer care near the end of life: Is it a quality-of-care issue? J Clin Oncol. 2008; 26(23):3860-3866.

57. Mack JW, Weeks JC, Wright AA, Black SD, Prigerson HG. End-of-life discussions, goal attainment, and distress at the end of life: predictors and outcomes of receipt of care consistent with preferences. J Clin Oncol. 2010;28(7):1203-1208.

58. Zhang B, Wright AA, Huskamp HA, et al. Health care costs in the last week of life - associations with end-of-life conversations. Arch Intern Med. 2009;169(5):480-488.

59. Steinhauser KE, Christakis NA, Clipp EC, et al. Factors considered important at the end of life by patients, family, physicians, and other care providers. JAMA. 2000;284(19):2476-2482.

60. Valdimarsdottir U, Helgason AR, Furst CJ, Adolfsson J, Steineck G. Awareness of husband's impending death from cancer and long-term anxiety in widowhood: a nationwide follow-up. Palliat Med. 2004;18(5): 432-443.

61. Hauksdottir A, Steineck G, Furst CJ, Valdimarsdottir U. Long-term harm of low preparedness for a wife's death from cancer - a population-based study of widowers 4-5 years after the loss. Am J Epidemiol. 2010;172(4):389-396.

62. Rainbird K, Perkins J, Sanson-Fisher R, Rolfe I, Anseline P. The needs of patients with advanced, incurable cancer. Br J Cancer. 2009;101(5): 759-764.

63. Degner LF, Kristjanson LJ, Bowman D, et al. Information needs and decisional preferences in women with breast cancer. JAMA. 1997; 277(18):1485-1492.

64. Hagerty RG, Butow PN, Ellis PA, et al. Cancer patient preferences for communication of prognosis in the metastatic setting. J Clin Oncol. 2004;22(9):1721-1730.

65. Lind SE, DelVecchio Good MJ, Seidel S, Csordas T, Good BJ. Telling the diagnosis of cancer. J Clin Oncol. 1989;7(5):583-589.

66. Butow PN, Maclean M, Dunn SM, Tattersall MHN, Boyer MJ. The dynamics of change: Cancer patients' preferences for information, involvement and support. Ann Oncol. 1997;8(9):857-863.

67. Heyland DK, Dodek P, Rocker G, et al; for Canadian Researchers End-of-Life Network (CARENET). What matters most in end-of-life care: perceptions of seriously ill patients and their family members. CMAJ. 2006;174(5):627-633

68. Parker PA, Baile WF, de Moor C, Lenzi R, Kudelka AP, Cohen L. Breaking bad news about cancer: patients' preferences for communication. J Clin Oncol. 2001;19(7):2049-2056. 
69. Butow PN, Dowsett S, Hagerty R, Tattersall MH. Communicating prognosis to patients with metastatic disease: what do they really want to know? Support Care Cancer. 2002;10(2):161-168.

70. Stewart DE, Wong F, Cheung AM, et al. Informational needs and decisional preferences among women with ovarian cancer. Gynecol Oncol. 2000;77(3):357-361

71. Clayton JM, Hancock KM, Butow PN, Tattersall MHN, Currow DC. Clinical practice guidelines for communicating prognosis and end-of-life issues with adults in the advanced stages of a life-limiting illness, and their caregivers. Med J Aust. 2007;186(12 Suppl):S77, S79, S83-S108

72. Stajduhar KI, Thorne SE, McGuinness L, Kim-Sing C. Patient perceptions of helpful communication in the context of advanced cancer. J Clin Nurs. 2010;19(13-14):2039-2047.

73. Curtis JR, Engelberg R, Young JP, et al. An approach to understanding the interaction of hope and desire for explicit prognostic information among individuals with severe chronic obstructive pulmonary disease or advanced cancer. J Palliat Med. 2008;11(4):610-620.

74. Agar M, Currow DC, Shelby-James TM, Plummer J, Sanderson C, Abernethy AP. Preference for place of care and place of death in palliative care: are these different questions? Palliat Med. 2008;22(7):787-795.

75. Clayton JM, Butow PN, Arnold RM, Tattersall MHN. Discussing end-of-life issues with terminally ill cancer patients and their carers: a qualitative study. Support Care Cancer. 2005;13(8):589-599.

76. Hinton J. An assessment of open communication between people with terminal cancer, caring relatives, and others during home care. J Palliat Care. 1998;14(3):15-23.

77. Hagerty RG, Butow PN, Ellis PM, et al. Communicating with realism and hope: incurable cancer patients' views on the disclosure of prognosis. J Clin Oncol. 2005;23(6):1278-1288.

78. Pardon K, Deschepper R, Stichele RV, et al; for EOLIC-Consortium. Preferences of patients with advanced lung cancer regarding the involvement of family and others in medical decision-making. $J$ Palliat Med. 2010;13(10):1199-1203.

79. Goncalves F, Marques A, Rocha S, Leitao P, Mesquita T, Moutinho S. Breaking bad news: experiences and preferences of advanced cancer patients at a Portuguese oncology centre. Palliat Med. 2005;19(7): 526-531.

80. Ellerby JH, McKenzie J, McKay S, Gariepy GJ, Kaufert JM. Bioethics for clinicians: 18 Aboriginal cultures. CMAJ. 2000;163(7):845-850.

81. Blackhall LJ, Murphy ST, Frank G, Michel V, Azen S. Ethnicity and attitudes toward patient autonomy. JAMA. 1995;274(10):820-825.

82. Back AL, Anderson WG, Bunch L, et al. Communication about cancer near the end of life. Cancer. 2008;113(7 Suppl):1897-1910.

83. Anderson WG, Alexander SC, Rodriguez KL, et al. "What concerns me is ..." Expression of emotion by advanced cancer patients during outpatient visits. Support Care Cancer. 2008;16(7):803-811.

84. Pollack KI, Arnold RM, Jeffreys AS, et al. Oncologist communication about emotion during visits with patients with advanced cancer. J Clin Oncol. 2007;25(36):5748-5752.

85. Alexander SC, Pollak KI, Moran PA, et al. How do non-physician clinicians respond to advanced cancer patients' negative expressions of emotions? Support Care Cancer. 2011;19(1):155-159.

86. Clayton J, Butow P, Arnold R, Tattersall M. Discussing life expectancy with terminally ill cancer patients and their carers: a qualitative study. Support Care Cancer. 2005;13(9):733-742.
87. Robinson TM, Alexander SC, Hays M, et al. Patient-oncologist communication in advanced cancer: predictors of patient perception of prognosis. Support Care Cancer. 2008;16(9):1049-1057.

88. Clayton JM, Butow PN, Arnold RM, Tattersall MHN. Fostering coping and nurturing hope when discussing the future with terminally ill cancer patients and their caregivers. Cancer. 2005;103(9):1965-1975.

89. Casarett D, Pickard A, Fishman JM, et al. Can metaphors and analogies improve communication with seriously ill patients? J Palliat Med. 2010;13(3):255-260.

90. McGrath P, Ogilvie KF, Rayner RD, Holewa HF, Patton MA. The "right story" to the "right person": communication issues in end-of-life care for Indigenous people. Aust Health Rev. 2005;29(3):306-316.

91. Weeramanthri T. "Painting a Leonardo with finger paint": medical practitioners communicating about death with aboriginal people. Soc Sci Med. 1997;45(7):1005-1015.

92. Strasser F, Palmer JL, Willey J, et al. Impact of physician sitting versus standing during inpatient oncology consultations: patients' preference and perception of compassion and duration. A randomized controlled trial. J Pain Symptom Manage. 2005;29(5):489-497.

93. Bruera E, Palmer JL, Pace E, et al. A randomized, controlled trial of physician postures when breaking bad news to cancer patients. Palliat Med. 2007;21(6):501-505.

94. Sapir R, Catane R, Kaufman B, et al. Cancer patient expectations of and communication with oncologists and oncology nurses: the experience of an integrated oncology and palliative care service. Support Care Cancer. 2000;8(6):458-463.

95. Bruera E, Pituskin E, Calder K, Neumann CM, Hanson J. The addition of an audiocassette recording of a consultation to written recommendations for patients with advanced cancer: a randomized, controlled trial. Cancer. 1999;86(11):2420-2425.

96. Clayton JM, Butow PN, Tattersall MH, et al. Randomized controlled trial of a prompt list to help advanced cancer patients and their caregivers to ask questions about prognosis and end-of-life care. J Clin Oncol. 2007;25(6):715-723.

97. Fallowfield L, Jenkins V, Farewell V, Saul J, Duffy A, Eves R. Efficacy of a Cancer Research UK communication skills training model for oncologists: a randomized controlled trial. Lancet. 2002;359(9307): 650-656.

98. Fallowfield L, Jenkins V, Farewell V, Solis-Trapals J. Enduring impact of communication skills training: results of a 12 month follow up. Br J Cancer. 2003;89(8):1145-1149.

99. Baile WF, Buckman R, Lenzi R, Glober G, Beale EA, Kudelka AP. SPIKES - a six-step protocol for delivering bad news: application to the patient with cancer. Oncologist. 2000;5(4):302-311.

100. Back AL, Arnold RM, Baile WF, et al. Efficacy of communication skills training for giving bad news and discussing transitions to palliative care. Arch Int Med. 2007;167(5):453-460.

101. Moore PM, Wilkinson SSM, Rivera Mercado S. Communication skills training for health care professionals working with cancer patients and their families and/or carers. Cochrane Database Syst Rev. 2004;(2): CD003751.pub2.

102. Andrade AD, Bagri A, Zaw K, Roos BA, Ruiz JG. Avatar-mediated training in the delivery of bad news in a virtual world. J Palliat Med. 2010;13(12):1415-1419.

103. Barnett MM. Effect of breaking bad news on patients' perceptions of doctors. J R Soc Med. 2002;95(7):343-347.
Cancer Management and Research

\section{Publish your work in this journal}

Cancer Management and Research is an international, peer-reviewed open access journal focusing on cancer research and the optimal use of preventative and integrated treatment interventions to achieve improved outcomes, enhanced survival and quality of life for the cancer patient The journal welcomes original research, clinical \& epidemiological

\section{Dovepress}

studies, reviews \& evaluations, guidelines, expert opinion \& commentary, case reports \& extended reports. The manuscript management system is completely online and includes a very quick and fair peerreview system, which is all easy to use. Visit http://www.dovepress.com/ testimonials.php to read real quotes from published authors. 